\section{A brake from the norm}

Calcium influx can be enhanced at presynaptic terminals by the positive coupling of calcium channels and calciumactivated potassium channels. In their report of this finding, $\mathrm{Xu}$ and Slaughter describe possible ways in which potassium channel activation can lead, somewhat unexpectedly, to the amplification of synaptic transmission.

Large-conductance calcium-activated potassium (BK) channels are present at presynaptic terminals throughout the nervous system, along with voltage-dependent calcium channels, the opening of which leads to neurotransmitter release. The $\mathrm{BK}$ channel is activated by calcium entry into the cell and by membrane depolarization. This hyperpolarizing channel would normally be expected to provide negative feedback to transmitter release, but there is evidence to suggest that this is not always the case.

One way in which BK channels might affect transmitter release is by prolonging action potential repolarization, but studies of the action potential waveform have failed to solve the conundrum of how BK channels influence synaptic transmission. To assess the role of BK channels in the absence of action potentials, $\mathrm{Xu}$ and Slaughter examined release at ribbon synapses of salamander rod photoreceptors, which are non-spiking.

In dark-adapted retinal slices, BK channel blockers were found to suppress light-evoked excitatory postsynaptic currents in second-order neurons by inhibiting transmitter release from rods. Xu and Slaughter went on to show that BK and calcium channels form a positive coupled loop at the presynaptic terminal: calcium influx activates the BK channel, leading to potassium efflux, which enhances calcium channel activity. It seems that outward BK and inward calcium currents approximately balance in the normal physiological voltage range of rods, allowing amplification of synaptic transmission to occur without a change in membrane voltage. However, when the rod is further depolarized, the BK current acts as a safety brake, overwhelming other currents and hyperpolarizing the cell.

The researchers suggest that a low-affinity potassiumbinding site on the extracellular side of the calcium channel enhances the calcium current, and that an increase in the concentration of local extracellular potassium could depolarize the rod terminal, thereby facilitating transmitter release. It remains to be seen whether a similar mechanism applies to some spiking neurons, in which BK channel blockers have also been found to suppress transmitter release.

Rebecca Craven

\section{(1) References and links}

ORIGINAL RESEARCH PAPER Xu, J. W. \& Slaughter, M. M. Large-conductance calcium-activated potassium channels facilitate transmitter release in salamander rod synapse. J. Neurosci. 25, 7660-7668 (2005)

FURTHER READING $\mathrm{Hu}$, $\mathrm{H}$. et al. Presynaptic $\mathrm{Ca}^{2+}$-activated $\mathrm{K}^{+}$channels in glutamatergic hippocampal terminals and their role in spike repolarization and regulation of transmitter release. J. Neurosci. 21, 9585-9597 (2001) | Pattillo, J. M. et al. Contribution of presynaptic calcium-activated potassium currents to transmitter release regulation in cultured Xenopus nerve-muscle synapses. Neuroscience 102, 229-240 (2001)

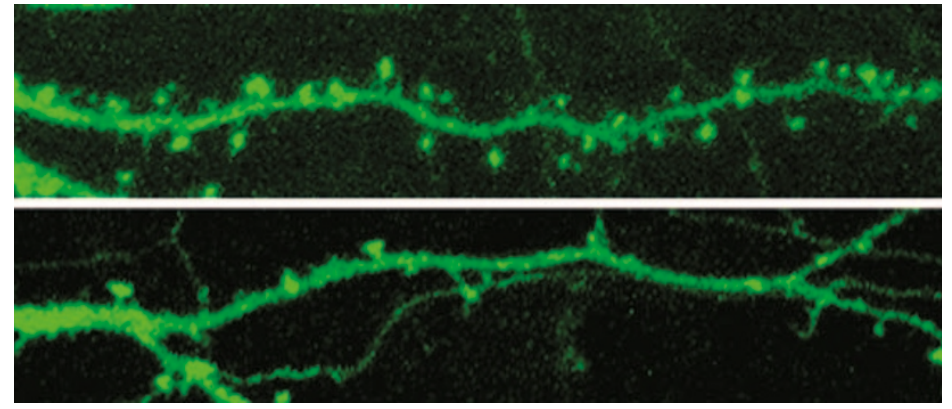

Hippocampal neurons develop numerous dendritic spines along their dendritic shafts during two weeks in culture (top). Silencing of one of the ORC subunits, ORC3, with siRNA, leads to a dramatic loss of dendritic spines in treated neurons (bottom).

\title{
CELL BIOLOGY OF THE NEURON
}

\section{Displaced connection}

The origin recognition complex (ORC), which is responsible for regulating DNA replication during the cell cycle, has a curious role in postmitotic neurons. Writing in the Journal of Cell Biology, Huang and colleagues show that the ORC subunits are expressed in the adult mouse brain and are crucial for dendrite and spine development.

The ORC is a hexameric protein that acts as a cyclin-dependent kinase (CDK)-regulated, ATPase-dependent molecular switch for initiating DNA replication during the cell cycle, ensuring that each wave of CDK activation is translated into one, and only one, round of genome replication. Adult neurons are mostly postmitotic and have no need for DNA replication, so it was a surprise when Huang et al. found that the Orc genes appeared frequently in the adult mouse brain database.

To explore their finding, the researchers studied Orc expression and found that the Orc2-6 mRNAs were abundant in adult brain tissues such as the cerebral cortex, hippocampus and cerebellum. Interestingly, Orc3 is expressed in the cytoplasm of cultured hippocampal neurons, rather than in the nucleus as one would expect, and the expression colocalizes with the dendritic marker microtubule-associated protein 2 (MAP2).

What is the function of ORC in postmitotic neurons? To address this issue, Huang et al. silenced Orc expression in cultured hippocampal neurons using small interfering RNAs
(siRNAs). Cultured hippocampal neurons undergo two stages of development: the initial arborization of dendrites at about 7-10 days in vitro (DIV) followed by the elaboration of spines at about 14-17 DIV.

In hippocampal neurons, silencing either Orc3 or Orc5 expression at 7 DIV results in the development of shorter dendrites with severely reduced branching. Similar manipulation of Orc expression at 14 DIV leads to a dramatic decrease in the density of spines along dendrites. These results suggest that the ORC is necessary for dendrite and spine development.

Three of the ORC subunits — ORC1, ORC4 and ORC5 - belong to a family of ATPases. It seems that the ORC's ATPase activity negatively regulates dendrite elaboration, as hippocampal neurons overexpressing a mutant ORC4 with defective ATP binding and hydrolysis show a significant increase in dendritic branching.

This newly discovered role of the ORC in dendrite and spine development is intriguing. The ORC might be a key part of the molecular machinery that translates patterns of neural activity into patterns of neuronal connectivity, a process that underlies long-term plasticity and memory.

(2) References and links Jane Qiu

ORIGINAL RESEARCH PAPER Huang, Z. et al. The origin recognition core complex regulates dendrite and spine development in postmitotic neurons. J. Cell Biol. 170, 527-535 (2005) FURTHER READING Bell, S. P. The origin recognition complex: from simple origins to complex functions. Genes Dev. 16, 659-672 (2002) 